
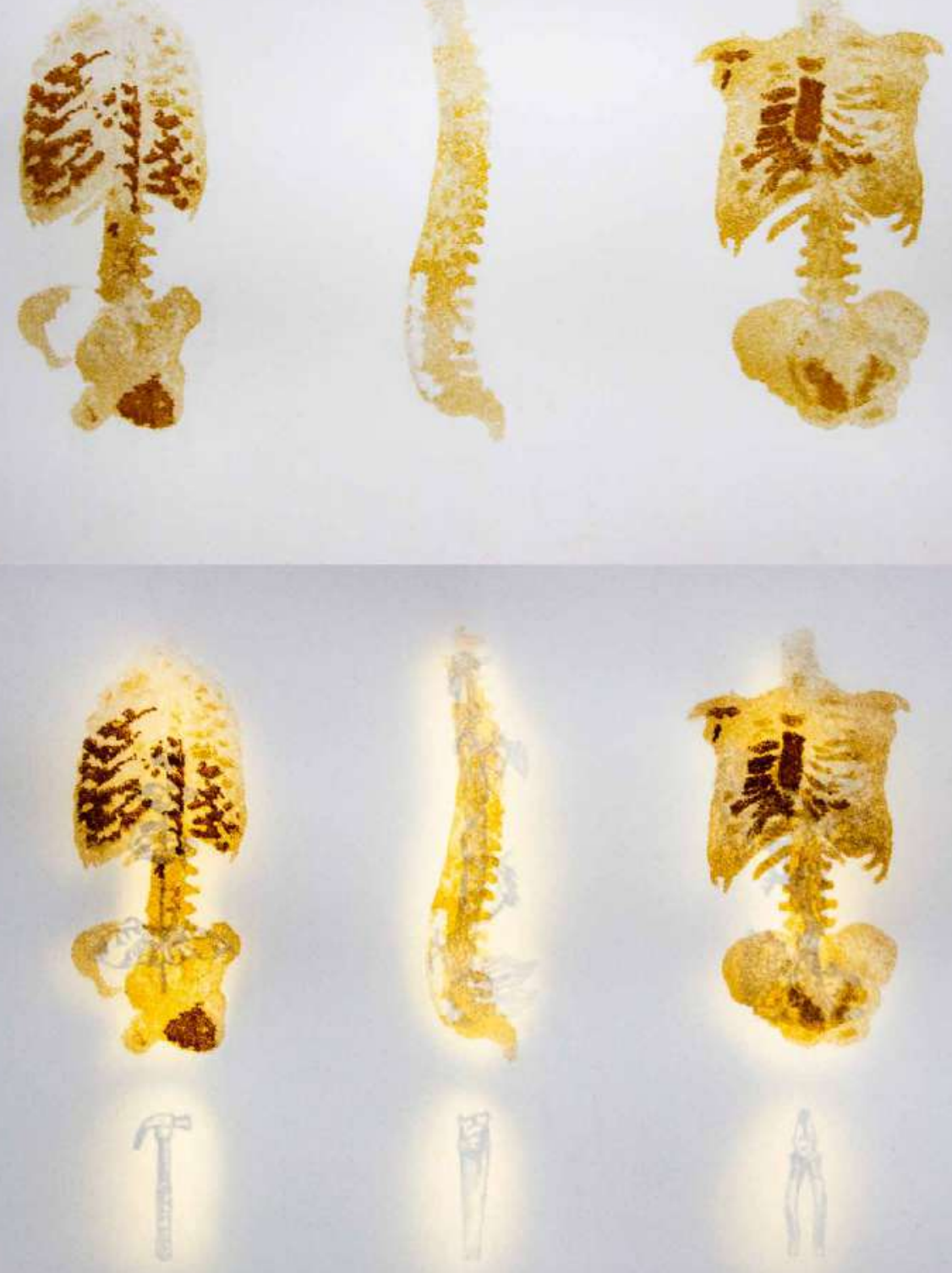

Yamith Quiroz David

Estudio 2

De la serie Herrumbres, cuerpos desechos

Dibujo con óxido ferroso, tinta, luz led cálida sobre papel Arches

$33,5 \times 43,5 \times 7 \mathrm{~cm}$

2020

Medellín 


\title{
Excombatientes, legados de violencia y competitividad electoral. Elecciones locales en Colombia, 2019*
}

\author{
Jose Antonio Fortou (Colombia)** \\ Santiago Sosa (Colombia) ${ }^{* * *}$
}

\section{Resumen}

Las elecciones de 2019 en Colombia fueron las primeras elecciones locales en las que el partido de excombatientes de las FARC participó. Dada la gran controversia alrededor de la firma e implementación del Acuerdo de Paz, así como la continuidad de la violencia en varios municipios, existe gran preocupación sobre la participación de los excombatientes. En este artículo se analiza la relación entre la participación de FARC y la competitividad de las elecciones para Alcaldía y Concejo a través de un análisis estadístico. Se encontró que los municipios en que la FARC participó presentaron mayor competitividad electoral, pero esta relación depende de los niveles recientes de violencia de cada municipio. La implicación principal de este análisis es que el legado de la violencia, no la participación de excombatientes, es el factor que debería primar en las preocupaciones sobre la contienda electoral.

\section{Palabras clave}

Elecciones; Partidos Políticos; Política Local; Competitividad Electoral; Posconflicto; Colombia.

Fecha de recepción: abril de 2020 - Fecha de aprobación: agosto de 2020

\section{Cómo citar este artículo}

Fortou,JoseAntonioySosa, Santiago.(2020). Excombatientes, legadosdeviolencia y competitividad electoral. Elecciones locales en Colombia, 2019. Estudios Políticos (Universidad de Antioquia), 59, pp. 280-301. DOI: 10.17533/udea.espo.n59a12

\footnotetext{
* Agradecemos a Juan Pablo Milanese de la Universidad Icesi por compartir generosamente los datos electorales y su conocimiento sobre el tema; asimismo, a los participantes del taller de investigación del Departamento de Gobierno y Ciencias Políticas de la Universidad EAFIT. Los archivos necesarios para replicar los análisis de este artículo se encuentran en https://github.com/josefortou/excomb-ep

${ }^{* *}$ Politólogo. Magíster y doctor en Ciencia Política. Profesor auxiliar del Departamento de Gobierno y Ciencias Políticas, Universidad EAFIT, Colombia. Correo electrónico: jfortour@eafit.edu.co - Orcid: 0000-0002-3127-4288 - Google Scholar: https://scholar.google.com/citations?hl=es\&user=NJ2itoAA AAAJ

*** Negociador Internacional. Magíster en Negocios Internacionales. Magíster y doctor en Ciencia Política. Profesor auxiliar del Departamento de Gobierno y Ciencias Políticas, Universidad EAFIT, Colombia. Correo electrónico: ssosanor@eafit.edu.co - Orcid: 0000-0002-9647-3720 - Google Scholar: https://scholar.google.com/citations?hl=es\&user=_PKwRkEAAAAJ
} 


\title{
Former Combatants, Legacies of Violence, and Electoral Competitiveness. Local Elections in Colombia, 2019
}

\begin{abstract}
The 2019 Colombian elections were the first ones in which the party of the former FARC combatants ran for local office. Given the great controversy surrounding the signing and implementation of the Peace Agreement, as well as the continuing levels of violence in several municipalities around the Country, there has been great concern about the electoral participation of the members of this political party. This article shows a statistical analysis of the relationship between the participation of the FARC party and its competitiveness in mayor and city council elections. We found that municipalities in which the FARC party ran for office had more competitive elections, but that it depended on the levels of recent violence experienced in each municipality. The main implication of our analysis is that the legacy of violence, not the electoral participation of former combatants, should be the main factor of concern in the electoral process.
\end{abstract}

\section{Keywords}

Elections; Political Parties; Local Politics; Electoral Competitiveness; PostConflict; Colombia. 


\section{Introducción}

El 27 de octubre de 2019 se llevaron a cabo las elecciones regionales en Colombia. En estas elecciones los colombianos eligieron alcaldes, concejales, gobernadores y diputados en 1101 municipios y 32 departamentos (Registraduría, s. f.). Más allá de la importancia de las elecciones regionales para la democracia representativa y participativa, las elecciones de 2019 fueron aún más importantes por ser las primeras elecciones regionales en las que participó el partido Fuerza Revolucionaria Alternativa del Común (FARC), el cual fue creado en el marco del Acuerdo de Paz de 2016 y de la desmovilización de las Fuerzas Armadas Revolucionarias de ColombiaEjército del Pueblo (FARC-EP).

Estas elecciones no estuvieron exentas de la controversia alrededor de la implementación del Acuerdo de Paz. Muchos consideran que el acuerdo le dio muchos beneficios a los excombatientes, siendo el extremo una preocupación sobre «entregarle el país a las FARC» (El Espectador, 2015, septiembre 28), y que la participación de los excombatientes en las elecciones y la implementación del Acuerdo de Paz podría impactar negativamente en la vida democrática del país. El partido Centro Democrático sostuvo que los

[282] Acuerdos de Paz dejarían impunes a los excombatientes y les proveerían de curules aunque continuaran activos en el negocio del narcotráfico (Semana, 2019, agosto 29).

¿Qué tan fundadas son estas preocupaciones?, ¿qué tan competitivas fueron las elecciones locales en Colombia disputadas en 2019 dada la presencia de este nuevo actor político-electoral? En este artículo se analiza el impacto de diferentes factores de la implementación del Acuerdo de Paz — desde la participación del partido FARC hasta la presencia de Circunscripciones Especiales para la Paz (CEP) o de Programas de Desarrollo con Enfoque Territorial (PDET) - en la competitividad de las elecciones en el país. Para ello, se construyó una base de datos original de resultados electorales para Alcaldías y Concejos, características municipales y violencia armada para todos los municipios colombianos que tuvieron elecciones locales en 2019.

Los hallazgos muestran que la participación de FARC no tuvo un impacto significativo sobre la competitividad de las elecciones a Alcaldía y Concejo, medida a través del número efectivo de partidos y del margen de victoria obtenido por los partidos ganadores respecto al segundo ganador. En cambio, 
se sugiere que la relación entre participación de FARC y competitividad electoral en el ámbito municipal está mediada por la intensidad reciente del conflicto armado. Finalmente, se encontró que la competitividad electoral en municipios ubicados en zonas de Circunscripciones Especiales para la Paz (CEP), en Espacios Territoriales para la Capacitación y Reincorporación (ETCR) o con Programas de Desarrollo con Enfoque Territorial (PDET) no fue distinta de los demás municipios del país.

La contribución se ubica en el marco de una literatura cada vez más robusta sobre la particularidad de las elecciones regionales en Colombia, su relación con el conflicto y la participación electoral de excombatientes; adicionalmente, se derivan unas expectativas empíricas; posteriormente, se evalúan estas expectativas usando datos originales recolectados después de las elecciones para Alcaldía y Concejo de 2019. ${ }^{1}$ Se describen las fuentes de información y las principales variables del estudio. Usando estos datos, se estima una serie de modelos de regresión lineal con el fin de describir la relación entre la competitividad electoral en el ámbito municipal, por un lado, y variables asociadas al conflicto armado, el posacuerdo y la presencia del partido FARC, por el otro. Los hallazgos sugieren que, si bien la participación de FARC aumentó la competitividad en las elecciones, su impacto depende del legado de la violencia reciente que cada municipio experimentó.

\section{Elecciones locales y conflicto en Colombia}

Las elecciones de 2019 ofrecen un caso de estudio interesante, tanto por el potencial efecto de la participación del partido FARC como por sus resultados electorales. En términos de resultados, la competitividad electoral en el ámbito municipal exhibe una alta heterogeneidad. La gráfica 1 muestra la distribución de dos indicadores de competitividad para las elecciones de Alcaldía y Concejo en todos los municipios para los cuales hay datos disponibles en el sitio web de la Registraduría Nacional del Estado Civil (s. f.). El indicador NEP hace referencia al número efectivo de partidos electorales según la fórmula de Markku Laakso y Rein Taagepera (1979). ${ }^{2}$ Por otro lado, el indicador margen mide la diferencia entre la proporción de votos

\footnotetext{
${ }^{1}$ Los archivos necesarios para replicar los análisis de este artículo se encuentran en https://github.com/ josefortou/excomb-ep

${ }^{2}$ Para el caso de las elecciones de 2019, la correlación del NEP, según la fórmula de Laakso y Taagepera (1979) y usando la fórmula de Grigirii Golosov (2010), es $>0.900$, lo cual sugiere que son empíricamente equivalentes en esta muestra. Se eligió la versión clásica de Laakso y Taagepera por ser la más aceptada en la disciplina.
} 
totales obtenidos por el partido más votado y el segundo más votado en un municipio. Las elecciones para Concejo fueron más competitivas, con mayor número efectivo de partidos - 6.2- y menor margen de victoria $-6,3 \%$ promedio que las de Alcaldía — 2.75 y $13,6 \%$, respectivamente-.

Gráfica 1. Heterogeneidad en la competitividad electoral en el ámbito municipal, Alcaldía y Concejo.

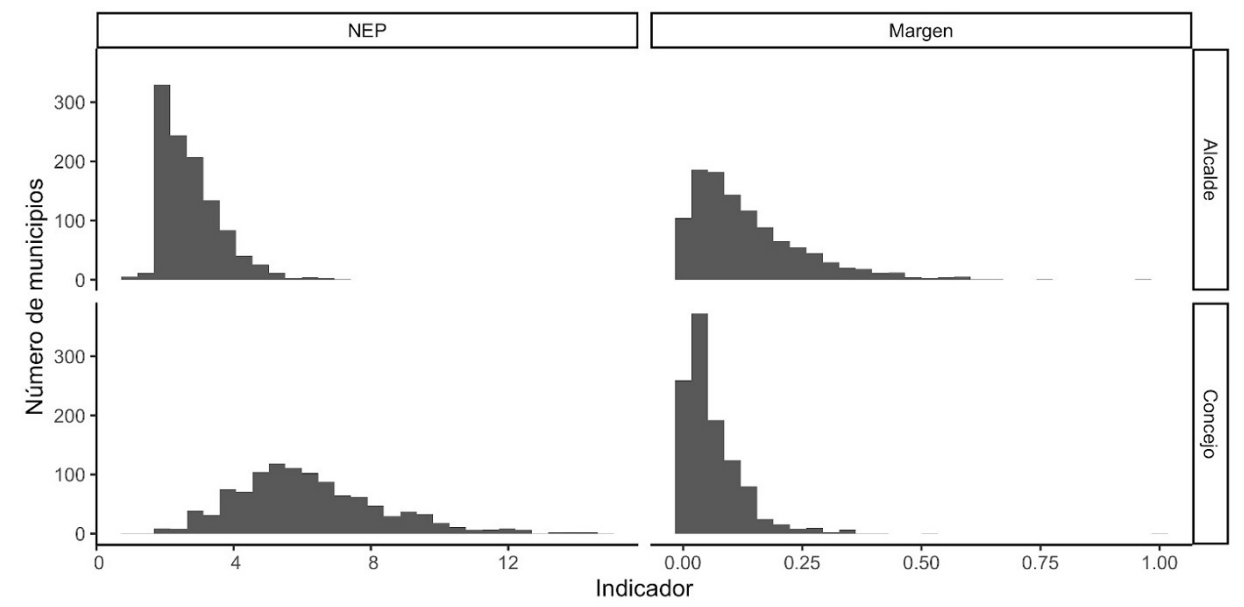

Fuente: elaboración propia a partir de Registraduría (s. f.).

¿Qué explica las diferencias en la competitividad electoral en el ámbito municipal en las elecciones de 2019 en Colombia? El estudio de las elecciones locales en Colombia despega con la apertura del sistema político en el ámbito subnacional, en particular, la elección popular de alcaldes en 1988. Los trabajos de Gustavo Bell y María De la Espriella (1988), Pilar Gaitán (1988) y de Manuel Alonso y William Pérez (1992) son ejemplos tempranos de estos estudios. Durante la década siguiente, otros se sumaron al análisis de sistemas de partidos y elecciones subnacionales, prestando especial énfasis a las municipales (Bejarano y Dávila, 1998; García Sánchez, 2000; Arenas y Escobar, 2000a; 2000b; Hoyos, 2005; García Oñoro y Godoy, 2009).

Esta área de estudios se fortalece tras la reforma política de 2003, la cual transformó el sistema electoral colombiano en todos los ámbitos: nacional, regional y local. Las compilaciones de Gary Hoskin y Miguel García (2006) y Rodrigo Losada y Patricia Muñoz (2007) evidencian un interés de los académicos colombianos por entender los cambios del sistema. Rápidamente, 
la unidad de análisis pasó de lo nacional a lo subnacional con trabajos como los de Lya Fernández (2003), Clara Rodríguez (2005), Miguel García (2006), Juan Pablo Ossa (2006), Claudia Buitrago (2007), Juan Camilo Vanegas (2008), Juan Carlos Arenas y John Fredy Bedoya (2011), Laura Wills y Margarita Batlle (2011), Juan Pablo Milanese y Luis Jaramillo (2015), José Antonio Fortou (2016), Juan Pablo Milanese, Adolfo Abadía y Luciana Manfredi (2016) y Juan Pablo Milanese, Adolfo Abadía, Alejandro Rodríguez y Beatriz Cuervo (2017), entre muchos otros. Algunos han mirado aspectos puntuales como la selección de candidatos (Batlle, 2012; Milanese y Barrero, 2016) o la desconexión local-nacional (Botero y Alvira, 2011). De esta manera, se ha consolidado una literatura especializada enfocada en los efectos de las reglas de juego sobre la competencia político-electoral en los ámbitos regional y local en Colombia.

En este artículo se aborda la relación entre competitividad electoral local, por un lado, y la participación de excombatientes en el marco del posconflicto, por el otro. Trabajos recientes han enfatizado esta conexión entre resultados electorales, política y conflicto en Colombia. Leopoldo Fergusson, James Robinson, Ragnar Torvik y Juan Vargas (2016) y Leopoldo Fergusson, Pablo Querubín, Nelson Ruiz y Juan Vargas (2020) muestran cómo las respuestas de políticos al conflicto están medidas por estrategia y resultados electorales, mientras que Daron Acemoglu, James Robinson y Rafael Santos (2013) demuestran los efectos de la presencia armada y de la violencia sobre las elecciones locales. Abbey Steele y Libia Schubiger (2018) dan un contexto general sobre la democracia electoral colombiana en el marco del conflicto y la descentralización de la competencia electoral. Camilo Nieto (2018) analiza las instancias de violencia y fraude electoral en el ámbito local, mostrando que la cercanía entre élites locales y grupos armados impacta negativamente en la calidad de la democracia electoral. En general, esta literatura encuentra que el conflicto armado impacta tanto las estrategias electorales, legales e ilegales, así como las actitudes de los votantes respecto a los partidos y a sus propuestas respecto a la situación de violencia.

A estas discusiones se le suma una novedad: la participación del partido FARC, el cual constituye un nuevo ejemplo de participación política de excombatientes en el marco de acuerdos de paz que la promueven (Matanock, 2017; Matanock y Staniland, 2018). La transición de grupos armados a partidos políticos no solo ha sido estudiada en el contexto colombiano (Boudon, 2001; Santofimio, 2007), sino que constituye ya una 
significativa veta en la literatura comparada en ciencia política (Acosta, 2014; Kovacs y Hatz, 2016; Matanock, 2016; Dresden, 2015; Manning y Smith, 2018; Daly, 2020).

Sin embargo, el hecho de que el Acuerdo de Paz se esté implementando en el país no elimina el efecto de la historia de violencia y conflicto a lo largo y ancho del país. Michael Weintraub, Juan Vargas y Thomas Flores (2015) evidencian que los legados de violencia armada durante el conflicto tuvieron un impacto significativo sobre la decisión de voto de los colombianos en la elección presidencial de 2014: municipios con niveles muy bajos o altos de violencia insurgente votaron menos por el entonces presidente Juan Manuel Santos.

Además, de acuerdo con Fergusson et al. (2020), la posibilidad de representación de grupos típicamente excluidos puede resultar en mayor violencia local debido a que las élites locales usan las herramientas a su disposición para defender su posición de poder. En ese sentido, las elecciones locales presentan un gran riesgo de violencia electoral respecto a partidos y grupos no tradicionales como FARC.

[286 ] ¿Qué efecto se espera que tenga la participación del partido FARC en las elecciones locales? La expectativa es que los municipios en los que participó FARC sean, en promedio, más competitivos que en los que no participó. Esto se debe a varios motivos: primero, la participación del partido FARC en una elección local, por definición, aumenta el número de partidos en contienda, lo cual aumentaría potencialmente la competitividad de las ciudades en las que se lanzan. Ahora bien, el hecho de que un partido o un movimiento se lance no necesariamente incrementa la competitividad, pues podrían tener un resultado electoral muy bajo y no obtener curules - un indicador como el número efectivo de partidos captura esto-.

Sin embargo, dado que el partido FARC es un partido recién formado y sin mucha fuerza electoral — comparados con otros más establecidos y dados sus resultados pobres en las elecciones presidenciales y legislativas de 2018, se esperaría que la decisión de FARC de competir en un municipio sea tomada estratégicamente, esto es, que compitan solamente en los municipios en donde la probabilidad esperada de obtener por lo menos una curul sea lo suficientemente alta. En efecto, la FARC compitió — solo o en coalición con otros partidos- en poco menos de $7 \%$ de las 2202 elecciones municipales —Alcaldía y Concejo- de los que se tienen datos. 
Así pues, si se asume que FARC eligió unos pocos municipios para competir de manera estratégica, entonces se esperaría que en esos municipios el número efectivo de partidos políticos aumente respecto a municipios similares en los que FARC no compitió. La primera hipótesis es, por tanto: $\mathrm{H}_{1}$ ceteris paribus, los municipios en donde FARC participó tienen en promedio un número efectivo de partidos mayor a los municipios en donde FARC no participó.

Segundo, la participación estratégica de FARC en las elecciones locales haría que la contienda fuera más difícil para los otros partidos. Así, si bien FARC no tiene un potencial electoral tan elevado como muchos otros partidos políticos colombianos, en donde decide participar, probablemente, tiene el suficiente potencial como para sustraer suficientes votos $-\mathrm{y}$, por tanto, potencial de obtener curules - a los demás, incluso a los partidos más preponderantes.

Por supuesto, esto no quiere decir que FARC pueda quitarles votos a todos los partidos, probablemente un adepto del Centro Democrático no votaría por FARC; sin embargo, es posible que el partido de FARC haya evitado municipios en los que el Centro Democrático domina. Como se señaló arriba, dado que FARC es un partido nuevo y con recursos limitados —en particular, en comparación con otros partidos-, entonces su elección de municipios en los cuales participar probablemente es un cálculo estratégico.

Así pues, si el partido FARC compitió donde consideró que podría tener un buen desempeño electoral, lo cual significa que otros partidos tendrían menor votación al perderla FARC si este partido obtiene un mínimo de votos, entonces se esperaría que fuera difícil «arrasar» en las elecciones. En otras palabras, se esperaría que el margen de victoria del partido ganador con respecto al segundo más votado fuera más pequeño, debido al incremento de la competitividad electoral que implica la participación de FARC. La segunda hipótesis es, por tanto: $\mathrm{H}_{2}$ ceteris paribus, los municipios en los que participó FARC presentan, en promedio, un menor margen de victoria del mayor partido respecto al segundo en comparación con municipios en los que FARC no participó.

Sin embargo, se debe considerar el legado del conflicto para entender las dinámicas electorales, especialmente cuando involucran la participación de excombatientes como integrantes de partidos políticos. Dadas las acciones 
de los grupos armados y del Estado durante el conflicto, no sería sorprendente que en algunos municipios hubiera escepticismo sobre FARC e incluso secuelas de intimidación de votantes y de riesgo electoral por parte de varios actores armados. El efecto de la participación de FARC en las elecciones locales depende, entonces, de la historia del conflicto en cada municipio. Por tanto, nuestra tercera hipótesis es: $\mathrm{H}_{3}$ la relación entre la participación de FARC y la competitividad de las elecciones en el ámbito municipal está mediada por la intensidad de la violencia reciente en cada municipio.

Estas hipótesis van en contravía de las preocupaciones expresadas por varios sectores de la política colombiana, incluyendo la derecha política. Como se señaló en la introducción, estos expresaban preocupación frente a la participación del partido creado por excombatientes de FARC cuando sugieren que allí donde participen la democracia electoral sufrirá. A continuación, se realiza un análisis estadístico de las elecciones locales con el fin de evaluar estas hipótesis y contribuir al debate académico en torno al tema.

\section{Diseño de investigación}

¿Qué tan competitivas fueron las elecciones locales en Colombia [288] disputadas en 2019? y iqué tanto influyó la participación del partido de excombatientes de FARC en estos resultados en el ámbito municipal? Para responder estas preguntas se realizó un análisis estadístico de una base de datos original construida a partir de diversas fuentes oficiales y académicas que incluye resultados electorales e información sobre conflicto armado en el ámbito municipal. Nuestra estrategia consiste en estimar una serie de modelos de regresión lineal que muestran la magnitud y la dirección positiva o negativa - de la asociación entre la participación electoral de FARC y la violencia reciente, por un lado, y los dos indicadores de competitividad electoral local, por el otro.

Se empezó por construir una base de datos original con el fin de evaluar la influencia del partido FARC en las elecciones locales de 2019, específicamente las de Alcaldía y Concejo. Para esto, se descargaron los resultados — preconteo- de estas dos elecciones en 1101 municipios directamente del sitio web de la Registraduría Nacional del Estado Civil (s. f.). Esto produjo 2202 observaciones —elecciones-municipio-. A partir de esta información, se calcularon dos variables que, juntas, permiten medir la competitividad electoral de las elecciones locales. 
Como medida de fragmentación electoral se calculó para cada municipio — para elecciones de Concejo y Alcaldía — el número efectivo de partidos (NEP) electoral según la fórmula de Laakso y Taagepera (1979). Asimismo, se calculó para las elecciones de Concejo y Alcaldía en cada municipio el margen de victoria, entendido como la diferencia entre la proporción de votos obtenidos por el partido más votado y la proporción de votos obtenidos por el segundo partido más votado. Estas dos medidas constituyen la operacionalización de la variable dependiente: la competitividad de las elecciones locales. Estas son las mismas variables discutidas en la gráfica 1.

Por otro lado, utilizando los mismos datos de la Registraduría Nacional (s. f.), se clasificó cada municipio y elección —Alcaldía y Concejo— según si el partido FARC, solo o en coalición con otros partidos, participó en los comicios presentando candidatos. Esto permite comparar municipios en donde FARC participó con aquellos en donde no lo hizo. Usando información oficial de la Agencia para la Reincorporación y la Normalización (ARN, s. f.), se clasificaron los municipios ubicados en Espacios Territoriales de Capacitación y Reincorporación (ETCR) y Programas de Desarrollo con Enfoque Territorial (PDET). Asimismo, se anotó si un municipio hace parte de una Circunscripción Especial para la Paz (CEP). Adicionalmente, utilizando información recopilada por el Centro de Estudios sobre Desarrollo Económico (CEDE) (Acevedo y Bornacelly, 2014), se calculó para cada municipio el número acumulado —en el periodo de 2015-2018 - de víctimas por homicidio incluidas en el Registro Único de Víctimas (RUV). Se argumenta que la participación electoral de FARC, la implementación de las instituciones formales del posconflicto PDET, ETCR y CEP - y el legado reciente de la violencia en el ámbito local pueden impactar la competitividad de las elecciones locales.

Finalmente, se incluyen otras características municipales con el fin de controlar explicaciones alternativas. Del sistema Terridata del Departamento Nacional de Planeación (DNP, s. f.), se tomó el indicador de medición del desempeño municipal (MDM) para 2017, el cual sirve como proxy de capacidad estatal local. La expectativa es que a mayor capacidad estatal más competitivas serán las elecciones, puesto que hay mayor seguridad en el proceso electoral. Finalmente, se incluyeron medidas del área municipal total —en kilómetros cuadrados_-, población total, distancia a Bogotá — en kilómetros - y distancia a la capital departamental —en kilómetros—. ${ }^{3}$

\footnotetext{
${ }^{3}$ Por su distribución, se calculó y utilizó el logaritmo natural de estas tres variables.
} 
Tomadas en conjunto, estas variables permiten controlar estadísticamente otros factores que influyen en el grado de la competencia partidista local.

\section{Resultados e interpretación}

¿Qué pasó en las elecciones realizadas en municipios donde el partido FARC participó?, ¿fueron más o menos competitivas? El partido FARC, de forma independiente o en coalición con otros partidos, compitió en apenas 72 de las 2202 elecciones municipales que se analizaron: esto incluye 13 candidatos para Alcaldía y 59 listas para Concejo. Esto puede deberse a una falta de capacidad organizacional para presentar candidatos ampliamente, una baja nacionalización del partido, consideraciones de corte estratégico o a una combinación de factores. En últimas, esta cifra evidencia que, cualquiera que sea el efecto de FARC sobre los resultados electorales, este está circunscrito a un número limitado de municipios.

Gráfica 2. Competitividad en los municipios donde el partido FARC hizo presencia electoral.

[290]

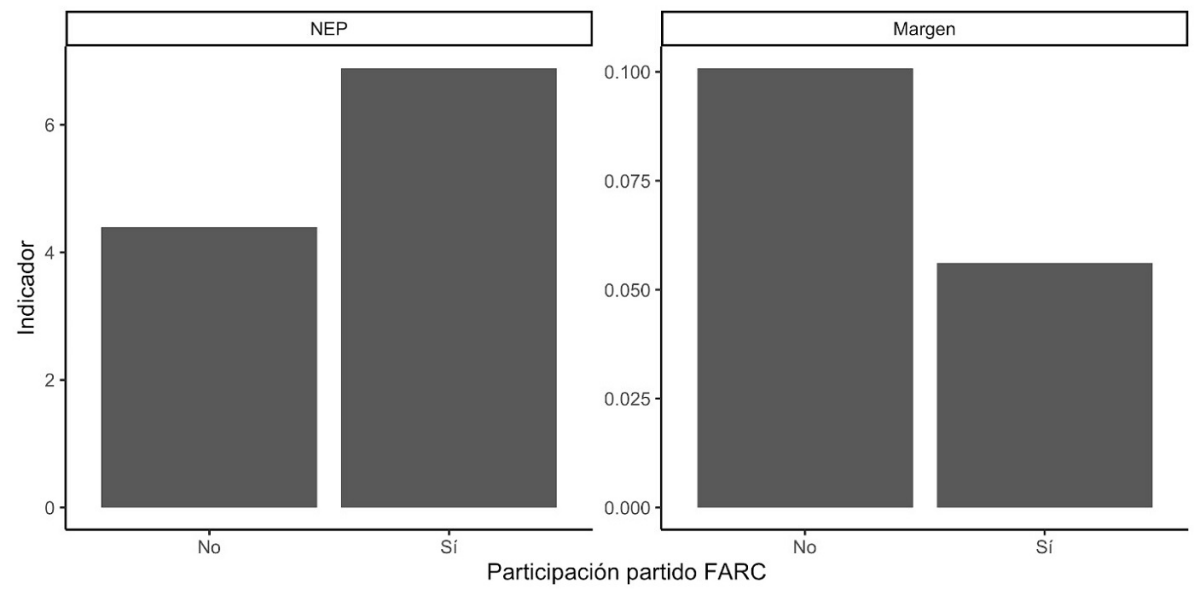

Fuente: elaboración propia a partir de Registraduría (s. f.).

Sin embargo, a primera vista hay diferencias significativas en el grado de competitividad de los municipios en donde el partido FARC hizo presencia electoral y aquellos en donde no. La gráfica 2 muestra el promedio del indicador de número efectivo de partidos electorales y del margen de victoria para elecciones a Alcaldía y Concejo, comparando municipios en 
donde el partido FARC participó con aquellos en que no. Allí donde sí hubo participación de FARC las elecciones fueron más competitivas: más partidos se presentaron y ganaron votos -6.9 partidos efectivos, en comparación con 4.4 en el resto del país- y las contiendas fueron más reñidas $-5,6 \%$ de margen de victoria, comparado con 10,1\% en otros municipios- Esto contradice visiones según las cuales la participación de FARC como partido sería negativa para la democracia local.

¿Es significativa esta diferencia entre municipios con participación de FARC y los demás?, ihay otros factores que una vez considerados hagan que esta relación sea espuria? Para ahondar en este primer hallazgo y responder estas preguntas, se estima una serie de modelos de regresión lineal, modelos en los que la variable dependiente es el NEP y, en otros, el margen de victoria. Para cada variable dependiente se estima un modelo de regresión lineal por mínimos cuadrados ordinarios con los datos agrupados — esto es, todas las observaciones - y luego se repete el análisis para las elecciones de Alcaldía y de Concejo por separado.

El foco está en los coeficientes de las variables de participación del partido FARC y la victimización homicida, los cuales indican la dirección y magnitud de la asociación entre estas variables y la competitividad de las elecciones; adicionalmente, se incluyó una interacción entre estas dos variables para evaluar la hipótesis 3 . En el contexto de un modelo de regresión lineal, una interacción es un término construido a partir de la multiplicación de dos variables y captura una relación de mediación o moderación, en otras palabras, ayuda a evaluar si la asociación estadística entre la participación del partido FARC y la competitividad en el ámbito municipal depende del número de víctimas por homicidio registradas en el RUV en años recientes — 2015-2018-.

La tabla 1 muestra los resultados de este análisis. Las primeras tres columnas muestran los resultados para modelos en los que la variable dependiente es el NEP electoral para todas las elecciones y luego para elecciones de Alcaldía y Concejo únicamente. Las últimas tres columnas repiten el ejercicio, pero cambia la variable dependiente por la otra medida de competitividad: el margen de victoria. Cada fila muestra el coeficiente para cada variable independiente, el cual indica la dirección —el signo- y la magnitud —el número- de la asociación con la variable dependiente. Entre paréntesis se indica el error típico y los asteriscos indican niveles de significancia — valores $p$ - A A final de la tabla 1 se incluye información general sobre cada modelo y su ajuste a los datos. 
Tabla 1. Efecto de la participación de FARC en las elecciones locales. Resultados de los análisis estadísticos.

\begin{tabular}{|c|c|c|c|c|c|c|}
\hline & $\begin{array}{l}\text { NEP } \\
\text { Todas }\end{array}$ & $\begin{array}{c}\text { NEP } \\
\text { Alcaldía }\end{array}$ & $\begin{array}{c}\text { NEP } \\
\text { Concejo }\end{array}$ & $\begin{array}{c}\text { Margen } \\
\text { todas }\end{array}$ & $\begin{array}{l}\text { Margen } \\
\text { Alcaldía }\end{array}$ & $\begin{array}{c}\text { Margen } \\
\text { Concejo }\end{array}$ \\
\hline Municipio PDET & $\begin{array}{c}0.424 \\
(0.585)\end{array}$ & $\begin{array}{c}0.018 \\
(0.468)\end{array}$ & $\begin{array}{c}0.800 \\
(0.980)\end{array}$ & $\begin{array}{l}-0.039 \\
(0.040)\end{array}$ & $\begin{array}{l}-0.036 \\
(0.070)\end{array}$ & $\begin{array}{l}-0.043 \\
(0.040)\end{array}$ \\
\hline Municipio ETCR & $\begin{array}{l}-0.088 \\
(0.221)\end{array}$ & $\begin{array}{c}0.123 \\
(0.178)\end{array}$ & $\begin{array}{l}-0.277 \\
(0.370)\end{array}$ & $\begin{array}{l}-0.001 \\
(0.015)\end{array}$ & $\begin{array}{l}-0.003 \\
(0.027)\end{array}$ & $\begin{array}{c}0.004 \\
(0.015)\end{array}$ \\
\hline Municipio CEP & $\begin{array}{l}-0.404 \\
(0.588)\end{array}$ & $\begin{array}{l}-0.156 \\
(0.471)\end{array}$ & $\begin{array}{l}-0.581 \\
(0.986)\end{array}$ & $\begin{array}{c}0.047 \\
(0.041)\end{array}$ & $\begin{array}{c}0.053 \\
(0.070)\end{array}$ & $\begin{array}{c}0.043 \\
(0.040)\end{array}$ \\
\hline Participación FARC & $\begin{array}{c}0.862^{* * *} \\
(0.199)\end{array}$ & $\begin{array}{c}0.093 \\
(0.256)\end{array}$ & $\begin{array}{l}0.827^{* *} \\
(0.267)\end{array}$ & $\begin{array}{l}-0.019 \\
(0.014)\end{array}$ & $\begin{array}{l}-0.037 \\
(0.038)\end{array}$ & $\begin{array}{l}-0.016 \\
(0.011)\end{array}$ \\
\hline $\begin{array}{l}\text { Víctimas por homicidio, } \\
\text { 2015-2018 }\end{array}$ & $\begin{array}{l}-0.004 \\
(0.005)\end{array}$ & $\begin{array}{l}-0.000 \\
(0.004)\end{array}$ & $\begin{array}{l}-0.007 \\
(0.008)\end{array}$ & $\begin{array}{l}0.001^{*} \\
(0.000)\end{array}$ & $\begin{array}{c}0.001 \\
(0.001)\end{array}$ & $\begin{array}{c}0.001 \\
(0.000)\end{array}$ \\
\hline FARC $x$ homicidio & $\begin{array}{c}-0.046^{* *} \\
(0.015)\end{array}$ & $\begin{array}{c}0.017 \\
(0.018)\end{array}$ & $\begin{array}{c}-0.093^{* * *} \\
(0.023)\end{array}$ & $\begin{array}{c}0.001 \\
(0.001)\end{array}$ & $\begin{array}{l}-0.000 \\
(0.003)\end{array}$ & $\begin{array}{l}0.002^{*} \\
(0.001)\end{array}$ \\
\hline Tipo de elección: Concejo & $\begin{array}{l}3.427^{* * *} \\
(0.060)\end{array}$ & & & $\begin{array}{l}-0.073^{* * *} \\
(0.004)\end{array}$ & & \\
\hline $\begin{array}{l}\text { Desempeño municipal, } \\
2017\end{array}$ & $\begin{array}{l}0.017^{* * *} \\
(0.004)\end{array}$ & $\begin{array}{l}0.007^{*} \\
(0.003)\end{array}$ & $\begin{array}{l}0.027^{* * *} \\
(0.006)\end{array}$ & $\begin{array}{l}0.001^{*} \\
(0.000)\end{array}$ & $\begin{array}{l}0.001^{*} \\
(0.000)\end{array}$ & $\begin{array}{c}0.000 \\
(0.000)\end{array}$ \\
\hline Población, log., 2019 & $\begin{array}{c}0.626^{* * *} \\
(0.034)\end{array}$ & $\begin{array}{c}0.198^{* * *} \\
(0.027)\end{array}$ & $\begin{array}{l}1.071^{* * *} \\
(0.058)\end{array}$ & $\begin{array}{c}-0.007^{* *} \\
(0.002)\end{array}$ & $\begin{array}{l}-0.005 \\
(0.004)\end{array}$ & $\begin{array}{c}-0.010^{* * *} \\
(0.002)\end{array}$ \\
\hline Área municipal, km. ${ }^{2}, \log$. & $\begin{array}{l}0.104^{* * *} \\
(0.031)\end{array}$ & $\begin{array}{c}0.102^{* * *} \\
(0.025)\end{array}$ & $\begin{array}{l}0.104^{*} \\
(0.051)\end{array}$ & $\begin{array}{l}-0.006^{* *} \\
(0.002)\end{array}$ & $\begin{array}{c}-0.010^{* *} \\
(0.004)\end{array}$ & $\begin{array}{l}-0.001 \\
(0.002)\end{array}$ \\
\hline Distancia a capital, log. & $\begin{array}{c}0.003 \\
(0.013)\end{array}$ & $\begin{array}{l}-0.013 \\
(0.010)\end{array}$ & $\begin{array}{c}0.012 \\
(0.021)\end{array}$ & $\begin{array}{c}0.000 \\
(0.001)\end{array}$ & $\begin{array}{l}-0.000 \\
(0.002)\end{array}$ & $\begin{array}{c}0.000 \\
(0.001)\end{array}$ \\
\hline Distancia a Bogotá, log. & $\begin{array}{l}-0.088^{*} \\
(0.036)\end{array}$ & $\begin{array}{c}-0.082^{* *} \\
(0.029)\end{array}$ & $\begin{array}{l}-0.114 \\
(0.062)\end{array}$ & $\begin{array}{c}0.001 \\
(0.003)\end{array}$ & $\begin{array}{c}0.003 \\
(0.004)\end{array}$ & $\begin{array}{l}-0.000 \\
(0.003)\end{array}$ \\
\hline $\mathrm{R}^{2}$ & 0.655 & 0.162 & 0.414 & 0.142 & 0.026 & 0.038 \\
\hline $\mathrm{R}^{2}$ ajust. & 0.653 & 0.150 & 0.406 & 0.135 & 0.011 & 0.024 \\
\hline Núm. obs. & 2201 & 1101 & 1100 & 2197 & 1097 & 1100 \\
\hline RECM & 1.405 & 0.795 & 1.663 & 0.097 & 0.119 & 0.068 \\
\hline
\end{tabular}

Fuente: elaboración propia. 
En líneas generales, los resultados indican que hay una asociación estadísticamente significativa entre la participación de FARC en el ámbito municipal y la competitividad medida por el NEP. Ceteris paribus, cuando se compara con el resto del país, los municipios con participación de FARC exhibieron en promedio 0.86 partidos efectivos más; sin embargo, estos resultados parecen ser dependientes del tipo de elección, pues la asociación entre la participación de FARC y el NEP en elecciones de Alcaldía no es distinta a cero.

A la inversa, al medimos la competitividad como margen de victoria hay una relación negativa entre esta variable y la participación de FARC, pero no es estadísticamente significativa. Es más, el modelo estadístico propuesto se ajusta mejor a las elecciones de Concejo que a las de Alcaldía, indicando que los factores que se asocian con la competitividad de estas últimas pueden ser otros. Así pues, hay evidencia parcial a favor de la hipótesis 1, según la cual los municipios en donde FARC participó exhiben mayor competitividad en términos del número efectivo de partidos.

El resultado más significativo es el coeficiente en la interacción entre la participación electoral del partido FARC y el acumulado de homicidios en cada municipio. Este término intenta capturar una intuición teórica importante: que la magnitud de la relación entre la participación de FARC en elecciones locales y la competitividad de estas depende de la severidad histórica —el legado_ de la violencia en el municipio. Los resultados de los modelos 1 y 3 dan soporte a esta hipótesis. Por un lado, en municipios sin participación de FARC, mayor violencia homicida no se asocia con un cambio en el número de partidos; por otro lado, en municipios en donde FARC sí participó, niveles históricos de violencia homicida se asocian con menos partidos efectivos.

Sin embargo, si bien los coeficientes de FARC y homicidios no son significativos en los modelos 4-6, el coeficiente de su interacción sí lo es en el modelo 6, aunque el efecto sustantivo — la magnitud del coeficiente- es muy pequeño. Hay, por tanto, poca evidencia en pro de la hipótesis 2. De otro lado, la significancia del coeficiente de la interacción entre FARC y homicidios en varios de los modelos da evidencia parcial a favor de la hipótesis 3.

Para ahondar en el análisis de la interacción entre la participación electoral de FARC y el legado de la violencia armada en el ámbito municipal, se utilizaron los modelos para elecciones a Concejo para realizar predicciones (King, Tomz y Wittenberg, 2000), comparando la asociación entre la 
participación electoral de FARC y la competitividad de las elecciones a Concejo para diferentes niveles de violencia. Las demás variables independientes están establecidas en sus medias y las variables binarias fueron establecidas en la categoría modal —municipios no PDET, CEP o ETCR—.

Se presentan los resultados en la gráfica 3. La subgráfica A muestra resultados basados en el modelo 3 -NEP para Concejo- y la subgráfica B basados en el modelo 6 -margen para Concejo-. Se estimaron e incluyeron intervalos de confianza de 95\%. Este ejercicio permite ver el valor esperado del grado de fragmentación electoral y del margen de victoria, dados distintos niveles de violencia, en municipios con y sin participación del partido FARC.

Gráfica 3. Relación entre la competitividad y la participación de FARC dependiendo del legado de violencia reciente.
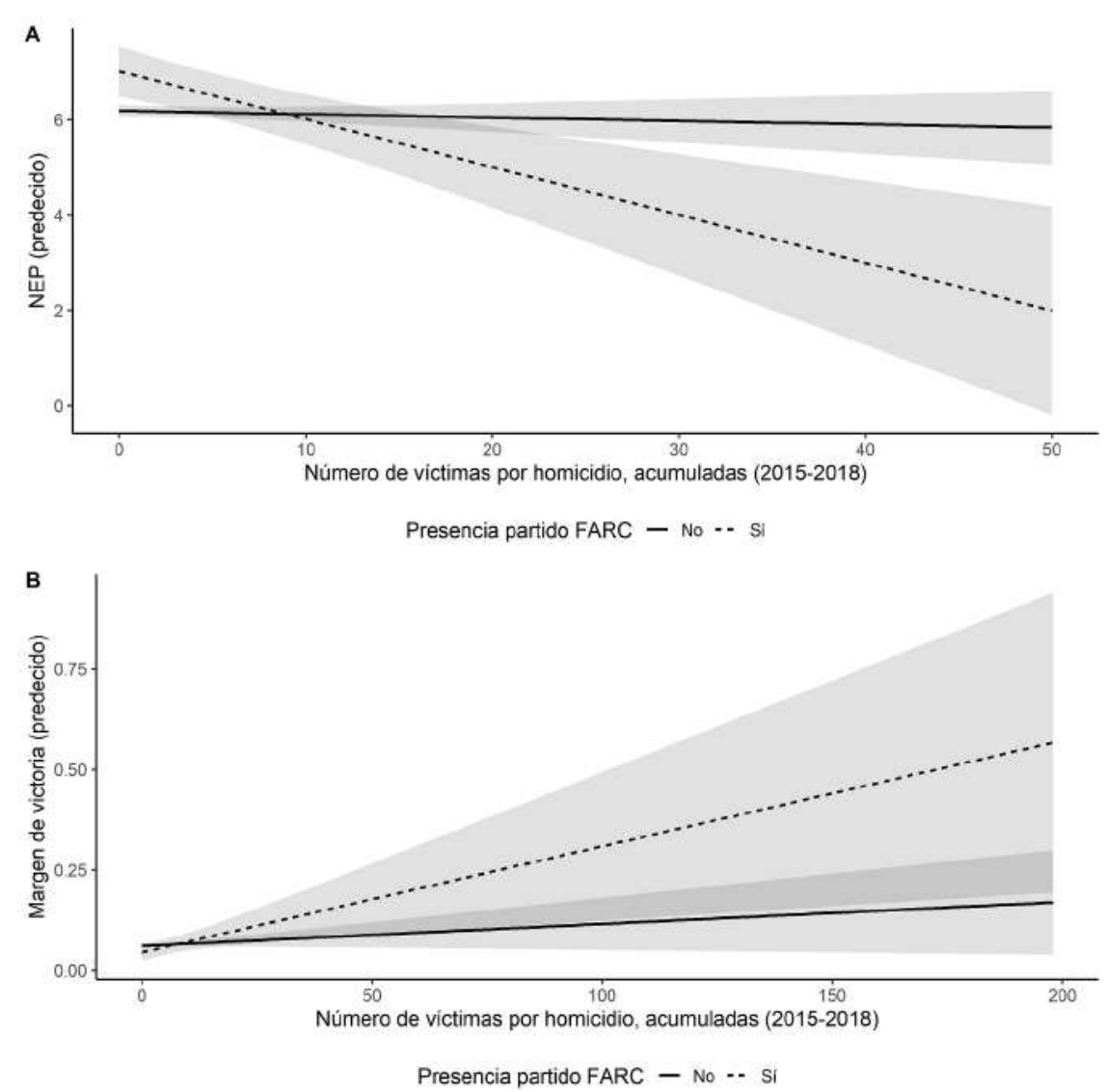

Fuente: elaboración propia. 
Como puede apreciarse —subgráfica A-, el impacto de la participación de FARC sobre el número efectivo de partidos depende del legado de violencia homicida reciente de cada municipio: mientras más homicidios acumulados hubo, menor es el NEP si FARC participó en la elección —línea punteada-. Por otro lado, el número de víctimas parece no afectar el NEP en los municipios en que FARC no participó —línea sólida-. Adicionalmente, se puede apreciar que el NEP es mayor en donde FARC participó y hubo pocos episodios de violencia. En términos concretos, en un municipio sin víctimas por homicidio reportadas en el RUV se espera ver entre 6 y 7 partidos efectivos, sin importar si FARC participa o no, pero en municipios con más violencia - 50 víctimas acumuladas en el periodo 2015-2018 - la diferencia en el número de partidos entre municipios con y sin participación es significativa —alrededor de 6 y 2 partidos efectivos, respectivamente-.

Respecto al margen de victoria — subgráfica B—, los municipios en los que participó FARC fueron menos competitivos si el legado de la violencia fue grande — línea punteada—, pero no hay cambios en el NEP asociados a un aumento en el número de víctimas en donde FARC no participó línea sólida-. Sin embargo, la diferencia entre los municipios en los que participó FARC y en los que no parece no ser estadísticamente significativa para casi la totalidad del rango de víctimas, tal y como lo muestran los intervalos de confianza.

En síntesis, el análisis basado en recolección de datos estadísticos y la estimación de una serie de modelos de regresión lineal sugiere que hay una relación positiva entre la participación del partido FARC en las elecciones locales y la competitividad de las elecciones, pero esta asociación depende de tres factores: primero, del aspecto de la competitividad que se observe, esta relación es más clara para el número de partidos electoral que para el margen de victoria; segundo, depende del tipo de elección, el modelo se ajusta mejor a los datos de elecciones de Concejo en comparación con los de Alcaldía; y tercero, la relación entre participación de FARC y competitividad depende del legado de violencia homicida reciente, pues a medida que aumenta el número de víctimas por homicidio disminuye el NEP y sube el margen de victoria en los municipios en donde participó FARC, pero no en donde este partido se abstuvo de presentar listas y candidatos. 


\section{Conclusión}

En este artículo se revisaron los resultados de las elecciones regionales de 2019, en particular, las de Alcaldía y Concejo, para entender mejor las diferencias en el grado de competitividad observadas en el ámbito local, dada la participación electoral del partido FARC.

Se encontró que la participación de FARC tiene un efecto positivo en la competitividad de las elecciones: los municipios en los que FARC participó tendieron a tener un número efectivo de partidos mayor y un margen de victoria menor. Sin embargo, dicho efecto está condicionado por la violencia sufrida en cada municipio en tiempos recientes: a mayor número de homicidios acumulados reportados en el RUV menos competitivas fueron las elecciones locales en los municipios en que FARC participó. Es decir, los municipios con mayores tasas de violencia y donde hubo participación del partido FARC presentan, en promedio, menor NEP y mayor margen de victoria. Este hallazgo concuerda con estudios recientes que encuentran que el comportamiento y actitudes políticas en el país dependen de factores relacionados con el conflicto armado y la exposición a la violencia (Gallego, 2018; Tellez, 2018).

Por tanto, no se encontró evidencia que sustente las preocupaciones de que la participación de las FARC en las elecciones locales haya tenido un impacto negativo en la competitividad de estas, es más, pudo haber tenido un efecto positivo sobre la competencia electoral local. Esto coincide con trabajos comparados que sugieren que la participación electoral de grupos militantes y excombatientes puede producir paces más duraderas (Matanock, 2017). En cambio, es el legado de la violencia armada lo que más ha impactado la competitividad electoral, lo que tiene una estrecha relación con la participación del partido FARC. Ello era de esperarse: probablemente muchos de los municipios en los que participaron fueron los mismos en los que más se sintió el conflicto armado.

Este último punto sugiere la pregunta sobre los determinantes de la participación de las FARC. En este artículo se asumió que FARC, debido a sus recursos limitados, escogió estratégicamente participar en lugares en que pudieran salir victoriosos con mayor probabilidad. Otros trabajos podrán enfocarse en la decisión de las FARC, es decir, analizar las características de los municipios que incentivan la participación de FARC. 
Para finaliza, se proponen cinco avenidas adicionales de investigación futura: primero, estudiar la relación entre las elecciones nacionales en que FARC ha participado - particularmente, las legislativas de 2018- y las elecciones de 2019; segundo, analizar por qué el partido FARC fue exitoso en unos municipios y no en otros; tercero, analizar el impacto de la participación de FARC en estas elecciones locales sobre el desarrollo de la política municipal, más allá de la competencia electoral; cuarto, realizar trabajos cualitativos que permitan una descripción profunda sobre las variaciones contextuales de los municipios en donde FARC participó para comprender los mecanismos causales a fondo; por último, llevar a cabo un análisis individual que considere abstencionismo, ideología e identidad partidista, y otro de partidos que considere los programas electorales y la selección de candidatos, entre otros, sería un complemento ideal al análisis aquí realizado.

\section{Referencias bibliográficas}

1. Acemoglu, Daron, Robinson, James A. \& Santos, Rafael. J. (2013). The Monopoly of Violence: Evidence from Colombia. Journal of the European Economic Association, 11 (1), pp. 5-44. https://doi.org/10.1111/j.1542-4774.2012.01099.x

2. Acevedo, Karina Margarita y Bornacelly, Iván David. (2014). Panel Municipal del CEDE. Bogotá, D. C.: Universidad de los Andes.

3. Acosta, Benjamin. (2014). From Bombs to Ballots: When Militant Organizations Transition to Political Parties. The Journal of Politics, 76 (3), pp. 666-683. https://doi. org/10.1017/S0022381614000188

4. Agencia para la Reincorporación y la Normalización (ARN). (s. f.). Agencia para la Reincorporación y la Normalización. Recuperado de http://www.reincorporacion. gov.co/es/reincorporacion/

5. Alonso Espinal, Manuel Alberto y Pérez Toro, William F. (1992). La elección popular de alcaldes y los mecanismos de poder político hoy: Guarne y Guatapé. Estudios Políticos, 1, pp. 91-110.

6. Arenas Gómez, Juan Carlos y Bedoya Marulanda, John Fredy. (2011). Las lógicas de la competencia electoral en escenarios locales: Medellín, 1988-2007. Estudios Políticos, 39, pp. 39-74.

7. Arenas Gómez, Juan Carlos y Escobar Escobar, Juan Carlos. (2000a). Discursos políticos y resultados electorales en Medellín durante los años noventas. Estudios Políticos, 16, pp. 73-99.

8. Arenas Gómez, Juan Carlos y Escobar Escobar, Juan Carlos. (2000b). Las elecciones de octubre en Medellín: candidaturas, temas y resultados. Estudios Políticos, 17, pp. 91-118. 
9. Batlle, Margarita. (2012). Selección de candidatos en las elecciones locales y regionales colombianas de 2011: Los casos de Huila y Santander. Reflexión Política, 14 (27), pp. 154-171.

10. Bejarano, Ana María y Dávila Ladrón De Guevara, Andrés. (1998). Elecciones y democracia en Colombia, 1997-1998. Bogotá, D. C.: Universidad de los Andes.

11. Bell Lemus, Gustavo Adolfo y De la Espriella, María Mercedes. (1988). 13 de marzo de 1988: Elección Popular de Alcaldes en Barranquilla. Candidatos, Campaña y Votación. Barranquilla: Universidad del Norte.

12. Botero, Felipe y Alvira, Daniel. (2011). Fulano de tal va por su aval. Desconexión entre los niveles nacionales y locales de los partidos políticos en Colombia. En: Wills Otero, Laura y Batlle, Margarita (eds.). Política y territorio: Análisis de las elecciones subnacionales en Colombia, 2011 (pp. 131-161). Bogotá, D. C.: PNUD, IDEA Internacional, NIMD.

13. Boudon, Lawrence. (2001). Colombia's M-19 Democratic Alliance: A Case Study in New-Party Self-Destruction. Latin American Perspectives, 28 (1), pp. 73-92. https://doi.org/10.1177/0094582X0102800105

14. Buitrago Restrepo, Claudia María. (2007). Los efectos de la reforma política y la Ley de Bancadas en el Concejo de Bogotá: Una corporación que se debate entre la nueva y la vieja política. Bogotá, D. C.: Uniandes.

15. Daly, Sarah Zukerman. (2020). Political Life after Civil Wars: Introducing the Civil War Successor Party Dataset. Journal of Peace Research. https://doi. [298] org/10.1177/0022343320920905

16. Departamento Nacional de Planeación (DNP). (s. f.). TerriData. Sistema de Estadísticas Territoriales. Descargas. Recuperado de https://terridata.dnp.gov.co/ index-app.html\#/descargas

17. Dresden, Jennifer Raymond. (2015). From Combatants to Candidates: Electoral Competition and the Legacy of Armed Conflict. Conflict Management and Peace Science, 34 (3), pp. 240-263. https://doi.org/10.1177/0738894215593676

18. El Espectador. (2015, septiembre 28). «Le van a entregar el país a las Farc por meterme a la cárcel»: Uribe al fiscal. Recuperado de https://www.elespectador. com/noticias/politica/le-van-entregar-el-pais-farc-meterme-carcel-uribe-al-fiarticulo-589358

19. Fergusson, Leopoldo; Querubín, Pablo; Ruiz, Nelson A. \& Vargas, Juan F. (2020). The Real Winner's Curse. American Journal of Political Science. https://doi. org/10.1111/ajps. 12508

20. Fergusson, Leopoldo; Robinson, James A.; Torvik, Ragnar \& Vargas, Juan F. (2016). The Need for Enemies. The Economic Journal, 126 (593), pp. 1018-1054. https://doi.org/10.1111/ecoj.12174

21. Fernández de Mantilla, Lya. (2003). El Comportamiento electoral en la elección de alcaldes 2003-Bucaramanga y su Área Metropolitana-Colombia. Avance de Investigación. Reflexión Política, 5 (10), pp. 152-169. 
Excombatientes, legados de violencia y competitividad electoral. Elecciones locales...

22. Fortou, Jose Antonio. (2016). Cambios en el sistema de partidos local de Medellín, 1988-2011. Estudios Políticos, 48, pp. 201-223. https://doi.org/10.17533/ udea.espo.n48a11

23. Gaitán, Pilar. (1988). Primera elección popular de alcaldes: expectativas y frustraciones. Análisis Político, 4, pp. 63-83.

24. Gallego, Jorge. (2018). Civil Conflict and Voting Behavior: Evidence from Colombia. Conflict Management and Peace Science, 35 (6), pp. 601-621. https://doi. org/10.1177/0738894218788362

25. García Oñoro, Jairo y Godoy, Horacio. (2009). Partidos, Movimientos y facciones en las elecciones populares de alcaldes en Barranquilla (Colombia), 19882007. Investigación y Desarrollo, 17 (1), pp. 62-83.

26. García Sánchez, Miguel. (2000). Elección popular de alcaldes y terceras fuerzas. El sistema de partidos en el ámbito municipal. 1988-1997. Análisis Político, 41, pp. 84-97.

27. García Sánchez, Miguel. (2006). La reforma electoral y su impacto en las elecciones locales del 2003: un análisis de las elecciones del Concejo de Bogotá. En: Hoskin Gary y García Sánchez, Miguel (eds.). La reforma política de 2003: ¿la salvación de los partidos políticos colombianos? (pp. 135-148). Bogotá, D. C.: Universidad de los Andes.

28. Golosov, Grigorii V. (2010). The Effective Number of Parties: A New Approach. Party Politics, 16 (2), pp. 171-192. https://doi.org/10.1177/1354068809339538

29. Hoskin, Gary y García Sánchez, Miguel (eds.). (2006). La reforma política de 2003: ¿la salvación de los partidos políticos colombianos? Bogotá, D. C.: Universidad de los Andes.

30. Hoyos, Diana. (2005). Evolución del sistema de partidos en Colombia, 19722000: Una mirada a nivel local y regional. Análisis Político, 18 (88), pp. 45-59.

31. King, Gary, Tomz, Michael \& Wittenberg, Jason. (2000). Making the Most of Statistical Analyses: Improving Interpretation and Presentation. American Journal of Political Science, 44 (2), pp. 347-361. https://doi.org/10.2307/2669316

32. Kovacs, Mimmi Söderberg \& Hatz, Sophia. (2016). Rebel-to-Party Transformations in Civil War Peace Processes 1975-2011. Democratization, 23 (6), pp. 990-1008. https://doi.org/10.1080/13510347.2016.1159558

33. Laakso, Markku \& Taagepera, Rein. (1979). «Effective» Number of Parties: A Measure with Application to West Europe. Comparative Political Studies, 12 (1), pp. 3-27. https://doi.org/10.1177/001041407901200101

34. Losada, Rodrigo y Muñoz Yi, Patricia. (2007). Las elecciones de 2006 en Colombia: una mirada desde la reforma política de 2003. Bogotá, D. C.: Pontificia Universidad Javeriana.

35. Manning, Carrie \& Smith, Ian. (2018). Electoral Performance by Post-Rebel Parties. Government and Opposition, 54 (3), pp. 415-453. https://doi.org/10.1017/ gov.2018.34 
36. Matanock, Aila M. (2016). Using Violence, Seeking Votes: Introducing the Militant Group Electoral Participation (MGEP) Dataset. Journal of Peace Research, 53 (6), pp. 845-853. https://doi.org/10.1177/0022343316668390

37. Matanock, Aila M. (2017). Bullets for Ballots: Electoral Participation Provisions and Enduring Peace after Civil Conflict. International Security, 41 (4), pp. 93-132. https://doi.org/10.1162/ISEC_a_00275

38. Matanock, Aila M. \& Staniland, Paul. (2018). How and Why Armed Groups Participate in Elections. Perspectives on Politics, 16 (3), pp. 710-27. https://doi. org/10.1017/S1537592718001019

39. Milanese, Juan Pablo y Barrero Escobar, Fredy A. (2016). Selección de candidatos en escenarios de atomización dirigencial: análisis de los casos de Cali y el Valle del Cauca, elecciones locales y regionales 2015. Análisis Político, 88, pp. 6989. https://doi.org/10.15446/anpol.v29n88.63602

40. Milanese, Juan Pablo y Jaramillo, Luis Eduardo. (2015). Impacto de los factores institucionales del sistema electoral en la fragmentación partidaria. Un análisis de las elecciones para concejos municipales en el Valle del Cauca (1997-2011). Colombia Internacional, 84, pp. 43-70. https://doi.org/10.7440/colombiaint84.2015.02

41. Milanese, Juan Pablo; Abadía, Adolfo A. y Manfredi, Luciana. (2016). Elecciones regionales en Colombia 2015: Análisis de las nuevas gobernaciones y asambleas departamentales. Revista Uruguaya de Ciencia Política, 25 (1), pp. 63-89.

42. Milanese, Juan Pablo; Abadía, Adolfo A.; Rodríguez, Alejandro y Cuervo, [300] Beatriz. (2017). Configuración de los apoyos electorales a nivel municipal. Un análisis de los resultados electorales para la Alcaldía de Cali, Colombia (2003-2015). Colombia Internacional, 90, pp. 67-98. https://doi.org/10.7440/colombiaint90.2017.03

43. Nieto-Matiz, Camilo. (2018). Democracy in the Countryside: The Rural Sources of Violence against Voters in Colombia. Journal of Peace Research, 56 (2), pp. 264-278. https://doi.org/10.1177/0022343318802986

44. Ossa, Juan Pablo. (2006). El efecto de la reforma política de 2003 sobre las corporaciones de representación popular: el caso del Concejo de Bogotá. Colombia Internacional, 64, pp. 182-191. https://doi.org/10.7440/colombiaint64.2006.09

45. Registraduría Nacional del Estado Civil. (s. f.). Elecciones 2019. Recuperado de http://elecciones1.registraduria.gov.co/pre_elec_2019/index.html\#/inicio/0/colombia

46. Rodríguez Pico, Clara Rocío. (2005). Reformas políticas y representación local en Bogotá. Estudios Políticos, 27, pp. 131-166.

47. Santofimio Ortiz, Rodrigo. (2007). La izquierda y el escenario político en Colombia: El caso de la participación política de la Unión Patriótica (UP) 1984-1986. Aspectos Preliminares sobre una Investigación. Virajes, 9, pp. 169-206.

48. Semana. (2019, agosto 29). Rearme de Márquez y Santrich, oxígeno para el uribismo. Recuperado de https://www.semana.com/nacion/articulo/rearme-de-lasfarc-fortalece-el-discurso-uribista/629660 
49. Steele, Abbey \& Schubiger, Libia I. (2018). Democracy and Civil War: The Case of Colombia. Conflict Management and Peace Science, 35 (6), pp. 587-600. https://doi.org/10.1177/0738894218787780

50. Tellez, Juan Fernando. (2018). Worlds Apart: Conflict Exposure and Preferences for Peace. Journal of Conflict Resolution, 63 (4), pp. 1053-1076. https:// doi.org/10.1177/0022002718775825

51. Vanegas Quintero, Juan Camilo. (2008). Impacto de la reforma política de 2003 en los partidos y en el sistema de partidos local. El caso de Cali, elecciones 2007. Perspectivas Internacionales, 4 (2), pp. 37-72.

52. Weintraub, Michael; Vargas, Juan F. \& Flores, Thomas E. (2015). Vote Choice and Legacies of Violence: Evidence from the 2014 Colombian Presidential Elections. Research \& Politics, 2 (2), pp. 1-8. https://doi.org/10.1177/2053168015573348

53. Wills Otero, Laura y Batlle, Margarita (eds.). (2011). Política y territorio: Análisis de las elecciones subnacionales en Colombia, 2011. Bogotá, D. C.: PNUD, IDEA Internacional, NIMD. 